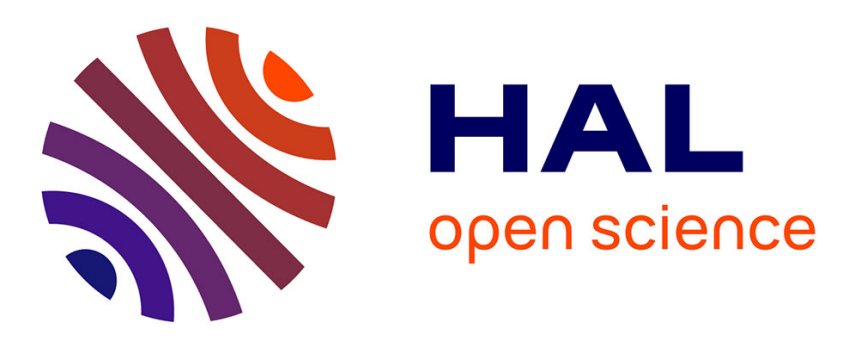

\title{
Feasibility and characterization of a safe susceptibility-matched endorectal coil for MR spectroscopy
}

Isabelle Saniour, Jean-Marie Verret, Cécile Rabrait-Lerman, Frank Pilleul, Olivier Beuf

\section{To cite this version:}

Isabelle Saniour, Jean-Marie Verret, Cécile Rabrait-Lerman, Frank Pilleul, Olivier Beuf. Feasibility and characterization of a safe susceptibility-matched endorectal coil for MR spectroscopy. NMR in Biomedicine, 2020, 33 (11), 10.1002/nbm.4384 . hal-02978666

\section{HAL Id: hal-02978666 \\ https://hal.science/hal-02978666}

Submitted on 26 Oct 2020

HAL is a multi-disciplinary open access archive for the deposit and dissemination of scientific research documents, whether they are published or not. The documents may come from teaching and research institutions in France or abroad, or from public or private research centers.
L'archive ouverte pluridisciplinaire HAL, est destinée au dépôt et à la diffusion de documents scientifiques de niveau recherche, publiés ou non, émanant des établissements d'enseignement et de recherche français ou étrangers, des laboratoires publics ou privés. 
Short title: Susceptibility-matched endorectal coil for MR spectroscopy spectroscopy 5220, U1206, F-69100, Lyon, France

3. Centre Léon Bérard, Department of Radiology, France

Corresponding author:

Olivier BEUF, PhD

CREATIS, CNRS UMR 5220, Inserm U1206

INSA Lyon, Bât Blaise Pascal (502)

7 Avenue Jean Capelle

F-69621 VILLEURBANNE

Tél. +33472438520 / 1597

olivier.beuf@creatis.insa-lyon.fr
Feasibility and characterization of a safe susceptibility-matched endorectal coil for MR

$$
\text { I. Saniour }{ }^{1} \text {, J.-M. Verret }{ }^{1,2} \text {, C. Rabrait-Lerman }{ }^{2} \text {, F. Pilleul }{ }^{3} \text {, O. Beuf }{ }^{1}
$$

1. Univ Lyon, INSA-Lyon, Université Claude Bernard Lyon 1, UJM-Saint Etienne, CNRS, Inserm, CREATIS UMR

2. General Electric Healthcare, Clinical Science Development Group, Buc, France

Keywords: Endorectal coil, susceptibility-matching material, RF heating 
When using endorectal coils, local radiofrequency (RF) heating may occur in the surrounding tissue. Furthermore, most endorectal coils create a susceptibility artifact detrimental to both anatomical magnetic resonance images (MRI) and spectroscopy (MRS) acquisitions. We aimed at assessing the safety and MRS performance of a susceptibility-matched endorectal coil for further rectal wall analysis. Experiments were performed on a GE MR750 3T unit. A variable number of miniaturized passive RF traps were incorporated in the reception cable. The assessment of RF heating and coil sensitivity was conducted on a $1.5 \%$ agar-agar phantom doped with $\mathrm{NaCl}$. Several susceptibility-matched materials such as Ultem, PFC, and barium sulfate were then compared with an external coil. Finally, Ultem was used as a solid support for an endorectal coil and compared with a reference coil. Phantom experiments exhibited a complete suppression of both the RF heating phenomenon and the coil sensitivity artifact. Ultem was the material that produced the smallest image distortion. The FWHM of MR spectra acquired using the susceptibility-matched endorectal coil showed at least $30 \%$ narrowing compared with a reference endorectal coil. A susceptibility-matched endorectal coil with RF traps incorporated was validated on phantoms. This coil appears to be a promising device for future in vivo experiments. 


\section{INTRODUCTION}

Colorectal cancer is the third most common cause of death among adult cancer patients in the United States ${ }^{1}$. The prognosis and the treatment course strongly depend on proper disease staging, which requires examination of the different layers of the rectal wall. For the initial staging of rectal cancer, endorectal ultrasound imaging is presently the most appropriate technique available, especially in the early T1 and T2 stages (following the Tumor Node Metastasis [TNM] classification). The assessment of local recurrence is particularly accurate with magnetic resonance imaging (MRI) phased array coils, but they have poor specificity and sensitivity in the early stages of colorectal cancer ${ }^{2}$. Indeed, the signal-to-noise ratio (SNR) provided by MR surface array coils is insufficient at clinical fields (1.5 $\mathrm{T}$ and $3 \mathrm{~T}$ ), and the achievable spatial resolution is, in this specific case, unsatisfactory. Since endorectal coils provide a significantly higher local signal sensitivity, higher spatial resolution may be achieved ${ }^{3}$. Endorectal MRI is thus believed to be a promising imaging examination.

The use of this type of coil has been controversial, however, because the signal distribution in the MR images may be inhomogeneous and the field of view (FOV) is limited. Artifacts caused by the coupling between the radiofrequency (RF) electric field and the conductive materials may affect images acquired with endorectal coils. These artifacts have been reported in the MRI literature as the "RF field non-uniformity." In fact, the reception cable, connecting the coil to the MR scanner, is sensitive to the electric component of the RF excitation field. Consequently, sheath currents are generated in the reception cable ground. At $3 \mathrm{~T}$, the wavelength $(\lambda)$ in air of the RF magnetic field for protons is $234 \mathrm{~cm}$; however, $\lambda$ depends on the electric permittivity of the medium and is given by:

$$
\lambda=\frac{c}{f}=\frac{c_{0}}{f \sqrt{\varepsilon_{r}}}=\frac{\lambda_{0}}{\sqrt{\varepsilon_{r}}}(1)
$$

where $c_{0}$ is the velocity $(\mathrm{m} / \mathrm{s}), f$ corresponds to the frequency $(\mathrm{Hz}), \lambda_{0}$ is the free space wavelength $(\mathrm{m})$, and $\varepsilon_{r}$ is the relative permittivity of the medium (without a unit).

First, this RF field non-uniformity will produce an inhomogeneous MR image thus altering the apparent coil sensitivity profile and leading to hypo-intense areas (black bands). Second, it will be the source of RF heating hazards in the surrounding tissues ${ }^{5,6}$. RF heat suppression circuits (such as 
1 RF traps) attenuate the amplitude of the electric currents circulating in the ground of the reception cable. Three architectures of RF traps are mainly used: conventional passive RF traps ${ }^{7,8}$, floating $\operatorname{traps}^{9}$, and active RF traps ${ }^{10}$. Floating and active RF traps are attractive and efficient techniques but more complicated to implement compared with passive traps. The passive RF trap is a widely used solution. Such circuits are easier to realize and potentially compact in size, but numerous traps need to be placed along the cable in order to achieve a complete suppression of RF heating. The location of the RF traps affects the efficiency of RF heat suppression, and they should be placed on the maxima of the standing wave circulating in the reception cable ${ }^{11}$.

To date, endorectal coils have been mainly evaluated for examinations of the prostate (prostate endorectal coil, PEC) or the rectal wall (colorectal endorectal coil, CEC). In vivo ${ }^{1} \mathrm{H}$ magnetic resonance spectroscopy (MRS) has shown to have clinical value in the diagnosis of brain, breast and prostate cancer. For instance, MRS provides relevant information on the metabolism of prostate cancer by locating and measuring levels of citrate, choline, creatine and polyamines ${ }^{12,13}$. The use of endorectal coils improve SNR and give access to higher spatial and temporal resolution that may be also useful for diagnosing and monitoring of Inflammatory Bowel Disease ${ }^{14}$.Clinical PECs are either flexible coils surrounded by a balloon inflated with air meant to prevent coil migration and unfold the loop coil (Medrad Prostate eCoil from Medrad or PMS Endo Coil Intera from Invivo) or rigid coils whose migration is prevented by a rigid arm (Sentinelle Endo Coil Array System from Hologic). Whereas clinical CEC migration is usually prevented by the organ itself (with the tightening of the colon ${ }^{14}$ ), manufactured coils are usually built with a plastic casing around an in-plane loop (or even two as is the case for the "Noras" two-channel endoanal coil) thus creating an air cavity ${ }^{15}$. A magnetic susceptibility artifact will then be created by the air-tissue interface between the coil and the surrounding tissues of the rectal wall. Depending on the geometry, a large difference in magnetic susceptibility between the two media can produce significant $B_{0}$ magnetic field inhomogeneity and magnetic field susceptibility gradients at the interface between the two media. The MRS is very sensitive to $B_{0}$ magnetic field inhomogeneity and thus requires susceptibilitymatching strategies ${ }^{16}$. A previous clinical study of patients with prostate cancer used a PEC inflated with a perfluorocarbon compound (PFC) instead of air and thereby significantly improved $\mathrm{B}_{0}$ uniformity showing a full width at half maximum (FWHM) reduced by a factor of 2 . Another study compared MRS examinations of the prostate with a PEC inflated with air, PFC, or barium sulfate 
1 suspension at $100 \% \mathrm{w} / \mathrm{v}$ and demonstrated a significant and similar decrease in the FWHM for PFC

2 and barium sulfate. The authors thus recommended barium sulfate since it is less expensive than

3 PFC ${ }^{17}$. Furthermore, PFC produces no visible signal on proton MR images whereas barium sulfate

4 produces low-intensity signal.

5 The present feasibility study aimed at characterizing a safe susceptibility-matched endorectal 6 coil for MR examination of the rectal or colonic wall. Temperature measurements and specific 7 absorption rate (SAR) calculations were performed to assess the safety of the endorectal coil. 8 Several miniaturized RF traps were built and incorporated in the reception cable to achieve efficient 9 RF heating reduction and a temperature increase of less than $1^{\circ} \mathrm{C}$, following the IEC-60601 10 requirements. The coil sensitivity pattern with and without traps was also compared.

11 Secondly, after a comparison of Ultem ${ }^{\mathrm{TM}}$ (Sabic, Riyadh, Saudi Arabia) with other susceptibility12 matched materials already described in the literature, a susceptibility-matched endorectal coil 13 incorporating Ultem ${ }^{\mathrm{TM}}$ material was built. Finally, the performance of this coil for spectroscopy 14 acquisitions was assessed using phantoms containing metabolite solutions. 


\section{Methods}

All experiments were performed on a GE MR750 $3 \mathrm{~T}$ system (General Electric Healthcare, Milwaukee, WI, USA) using a custom-made endorectal receiver coil (60 mm length, $6 \mathrm{~mm}$ width), as shown in Figure 1. A variable number of miniaturized passive RF traps, parallel LC circuit consists of a fixed inductor $(\mathrm{L}=200 \mathrm{nH})$ and a variable capacitor $(2-22 \mathrm{pF})$, were incorporated in the reception cable for RF heat suppression. We varied the capacitor until the trap was tuned to $128 \mathrm{MHz}$. The impedance of the trap was estimated using the S21 parameter with and without the trap. The trap adds an impedance of around $700 \mathrm{k} \Omega$.

\subsection{Safety of the endorectal coil: RF heating reduction}

\subsubsection{Experimental temperature measurements}

First, local temperature measurements were performed with and without miniaturized RF traps incorporated in the reception cable to assess RF heating reduction below the authorized level. The endorectal coil was placed in a cylindrical phantom (120 mm height and $200 \mathrm{~mm}$ diameter), as shown in Figure 2. It contained $1.5 \%$ agar-agar gel doped with $0.9 \% \mathrm{NaCl}$ (phantom 1), whose electrical conductivity was $\sigma=1 \mathrm{~S} / \mathrm{m}$, which is a suitable medium for heat propagation. Four fiber optic probes were taped on the endorectal coil only and not on the cable: three at the distal extremity and one at the proximal base of the coil and connected to a temperature measurement device $-0.1{ }^{\circ} \mathrm{C}$ resolution, $0.4 \mathrm{~Hz}$ sampling rate (Tempsens device from Opsens, Quebec, Canada). These locations were determined in accordance with previous studies demonstrating that heating concentrations occur at the distal extremities of a conductive object This assumption is applicable for small-sized elongated endorectal coils. Furthermore, as a preliminary step, temperature measurements were performed by moving the tip of the optical fiber along the distal extremity of the endorectal coil during the acquisition, until no superior temperature increase was found. Fastspin echo (FSE-XL) - the GE optimized version of fast-spin echo, which includes blurring cancellation for improved quality-axial images were acquired with the endorectal coil, first without any RF traps and then with five RF traps integrated into the reception cable. The cable path and cable length were kept the same.

The degree of RF heating depends on various parameters: the local electric fields induced by the energy transmitted to the phantom or tissue, the properties of the tissue, as well as the 
1 dimensions and geometry of the coil, the length of the cable and the position of the traps along the cable. The amplitude of the electric field is proportional to the amplitude of the RF magnetic field emitted at a constant duration. Indeed, a short TR produces a more intense time-averaged electric field. Moreover, the quadratic increase in temperature with the flip angle was confirmed experimentally by Armenean et al. ${ }^{5}$ in the case of True-FISP or FIESTA sequences. Hence, there is a tradeoff between flip angle and repetition time. In our experiments, TR was kept short to enable a high average electric field, and a $35^{\circ}$ flip angle was applied in order to avoid melting of the surrounding agar-agar gel and thus modification of its thermal properties.

For temperature measurements, the reception cable length, the cable path, the acquisition sequence, and the scan parameters were set for the worst-case scenario (the maximum heating). A FIESTA (equivalent to True-FISP or balanced FFE) sequence was used; it consists in a steady-state free precession sequence where gradients are balanced on all axes over a single TR. The parameters of the sequence were: acquisition time $=96 \mathrm{~s}$, flip angle $=35^{\circ}, \mathrm{FOV}=48 \times 48 \mathrm{~cm}^{2}$, matrix $=128 \times 128$, bandwidth $=250 \mathrm{kHz}$, and $T E / T R=0.99 \mathrm{~ms} / 2.7 \mathrm{~ms}$. The receiver cable was an RG58 coaxial cable whose length remained constant at $310 \mathrm{~cm}$ and which incorporated between 0 and $5 \mathrm{RF}$ traps. The RF traps were placed as shown in Figure 2. The trap bulk size was kept restricted: $7 \mathrm{~mm}$ outer diameter, which is the diameter of the air core inductance and only enlarges the RG58 cable diameter by $2 \mathrm{~mm}$. Inside the bore magnet, the receiver cable path passed through the maxima of the electric field. These maxima were located using an electric dipole linked to an LED HLMP 4015 (Agilent Technologies, Santa Clara, CA, USA) whose luminous intensity thus reflected the intensity of the electric field. Finally, the RF traps were shielded, in order to prevent them from receiving currents generated by the magnetic field. The shield consisted of a copper casing surrounding the RF trap. resulting in a near-null magnetic field inside the copper casing. This was realized by using a 0.1-mm copper tape wrapped around the body of the RF trap.

\subsubsection{Numerical estimations of SAR}

Based on temperature variation measurements, the local SAR values can be estimated using he a generic Bioheat Transfer Thermal Modal22. However, an agar-agar phantom was used for this experiment and there is no perfusion. Thus, the local SAR values were numerically estimated using a simplified bioheat equation and the parameters related to the perfusion and the metabolic heating were not considered: 


$$
\rho c \frac{\partial \Delta T}{\partial t}=\nabla \cdot(\kappa \nabla T)+\rho \cdot S A R-B\left(T-T_{B}\right)-A
$$

where SAR is in $\mathrm{W} / \mathrm{kg}, T$ is the temperature of the medium in ${ }^{\circ} \mathrm{C}, t$ is the time in $\mathrm{s}, \rho$ is the density in $\mathrm{kg} / \mathrm{m}^{3}, c$ is the specific heat, $\kappa$ is the thermal conductivity, $B$ is a coefficient related to blood perfusion, $\mathrm{T}_{\mathrm{B}}$ is the blood temperature in ${ }^{\circ} \mathrm{C}$, and $\mathrm{A}$ is the basal metabolic rate.

The numerical estimations were performed with Matlab R2012b (The MathWorks Inc., Natick, MA, USA) using the PDE toolbox. The same coil geometry previously described, and an agar-agar phantom (70 mm thickness, $200 \mathrm{~mm}$ diameter) were designed and imported to Matlab. The thermal properties of the agar-agar gel were assigned to the disk $\left(k=0.55 \mathrm{~W} /\left(\mathrm{m} .{ }^{\circ} \mathrm{C}\right), \rho=998 \mathrm{~kg} / \mathrm{m}^{3}, c=4200\right.$ $\left.\mathrm{J} /\left(\mathrm{kg} .{ }^{\circ} \mathrm{C}\right)\right)$. Using equation (2), the temperature distribution in an agar-agar phantom was simulated. The simulation was first performed with an arbitrarily high local SAR value $(100 \mathrm{~W} / \mathrm{kg})$ at the two proximal and distal sides of the coil where the temperature increase is the highest. The mesh element (the "hot spot") yielding the maximum temperature increase $\Delta T$ sim was selected and compared with the experimental temperature increase, $\Delta T \exp$. The estimation was then iterated with different values of local SAR, following a dichotomy process; the local SAR value was estimated. The input power is given by the lumped port that simulates the coaxial cable. The temperature distribution was simulated for a duration of between 0 and $360 \mathrm{~s}$ (temporal resolution of $0.01 \mathrm{~s}$ ). For each mesh element $(16,192$ triangular mesh elements covering the surface), the corresponding temperature increase was computed. The size of the mesh element was approximately $1.9 \mathrm{~mm}^{2}$ ensuring a tight mesh of the surface.

\subsection{Comparison of susceptibility-matched materials}

Several candidates for susceptibility matching were investigated in order to reduce the magnetic susceptibility artifact. The susceptibility of human tissues was considered close to water susceptibility (a classic assumption). Ultem ${ }^{T M}$ (i.e., Polyetherimide: $\mathrm{C}_{37} \mathrm{H}_{24} \mathrm{O}_{6} \mathrm{~N}_{2}$ ) is sometimes used as a plug for high-resolution NMR of liquids (susceptibility-matched sample plugs at Doty Scientific Inc., Columbia, SC, USA) and is a solid plastic with a second kind of magnetic susceptibility compatibility with human tissues. A comparison of different susceptibility-matched material was performed with Ultem ${ }^{\mathrm{TM}}$, air (no susceptibility-matched material), barium sulfate at $100 \% \mathrm{w} / \mathrm{v}$, and perfluorocarbon compound FC-72 $\left(\mathrm{C}_{6} \mathrm{~F}_{14}\right)$ so-called PFC. The consequent maximum $\mathrm{B}_{0}$ field 
1 inhomogeneity due to the magnetic susceptibility difference with water is $0.24 \mathrm{ppm}$ for Ultem ${ }^{\mathrm{TM}}$ versus $11.2 \mathrm{ppm}$ with barium sulfate or $2.3 \mathrm{ppm}$ with PFC. Ultem ${ }^{\text {TM }}$ thus theoretically creates a $\mathrm{B}_{0}$

field inhomogeneity much smaller than other susceptibility-matched materials especially at intermediate field. $B_{0}$ field inhomogeneities generate MR image distortions that can be measured as shifts in pixel position $\Delta x$. However, the same pixel shifts will be much more visible for an object of small dimensions than for an object of larger dimensions. Assuming a cylindrical shape, $\Delta \mathrm{x} / \mathrm{a}$ was computed, where $a$ is the radius of the cylinder creating the distortion (20). $\Delta x / a$ was equal to 2.70 for air, 2.02 for barium sulfate, 0.42 for PFC, and finally 0.035 for Ultem ${ }^{\mathrm{TM}}$.

MR images were acquired with an HD T/R knee array coil (GE Healthcare, Milwaukee, WI, USA) on a 60 -mm-diameter cylinder filled with saline water with $4.5 \mathrm{~g}$ of salt per liter of distilled water (phantom 2, Figure 3), thus simulating, from a magnetic susceptibility point of view, the human tissues which is usually considered within $15 \%{ }^{23}$ of the magnetic susceptibility of water. The central hole $\left(6 \mathrm{~mm}\right.$ diameter) allowed for the insertion of an Ultem ${ }^{\mathrm{TM}}$ cylinder $(6 \mathrm{~mm}$ diameter, $60 \mathrm{~mm}$ length) whose dimensions were identical to the endorectal coil. The peripheral holes $(5 \mathrm{~mm}$ diameter) were regularly placed (every $60^{\circ}$ ) around the central hole and facilitated the insertion of high-resolution NMR tubes. The tubes were located $1 \mathrm{~cm}$ away from the center of the phantom and contained air, water, barium sulfate, or PFC. Three locations labeled 1, 2, and 3 must be considered. Locations 1 and 2 housed the susceptibility-matched materials assessed (Ultem ${ }^{\mathrm{TM}}$, PFC, or barium sulfate). Location 3 housed an empty tube that serves as a reference for the air-water interface and describes the artifacts that appear when the magnetic susceptibility artifact is not considered.

The phantom axis was oriented orthogonally to the $B_{0}$ magnetic field; this orientation makes it possible to highlight the $\mathrm{B} 0$ inhomogeneity and therefore the magnetic susceptibility artifact. For a given susceptibility difference between two media and a given shape (cylindrical or other), artifact amplitudes will depend on the orientation of the object regarding the magnetic field $\mathrm{B}_{0}$. For a cylinder, it is maximal when the cylindrical axis is orthogonal to $B_{0}$. The phantom thus had an orthogonal orientation during the experiments. The difference in susceptibility between two media results in artifacts in the shape and signal intensity due to pixel shifts in the readout direction ${ }^{23}$. The pixel shifts occur in the readout direction (with the exception of echo planar imaging) and can be formulated as $\Delta x=\Delta B_{z}(x, y) / G_{R}$ with $G_{R}$ the readout gradient in the $x$ direction. The magnetic susceptibility artifact was maximized with a low readout gradient amplitude (narrow receiver 
1 bandwidth for a given FOV). The slice thickness was set to $2 \mathrm{~mm}$ to limit slice deformation during the selection process in the presence of inhomogeneity. The shim values were kept the same across all acquisitions in order to observe differences arising from the susceptibility modifications only. Finally, an FSE-XL sequence was acquired with TE/TR $=29.1 \mathrm{~ms} / 1500 \mathrm{~ms}$, echo train $=10$, slice thickness $=2 \mathrm{~mm}$, matrix $=320 \times 256, \mathrm{FOV}=70 \times 70 \mathrm{~mm}^{2}$, pixel bandwith $=27.1 \mathrm{~Hz} / \mathrm{px}$, and a gradient echo (GRE) sequence was acquired with TE/TR $=15.8 \mathrm{~ms} / 300 \mathrm{~ms}$, flip angle $=30^{\circ}$, slice thickness $=2 \mathrm{~mm}$, matrix $=512 \times 384, \mathrm{FOV}=80 \times 80 \mathrm{~mm}^{2}$, and pixel bandwith $=46.5 \mathrm{~Hz} / \mathrm{px}$.

\subsection{MRS using a susceptibility-matched endorectal coil}

Having tested different susceptibility-matched materials, an endorectal coil incorporating Ultem $^{\mathrm{TM}}$ was compared with an endorectal coil without filling material and referenced as "standard." The standard endorectal coil encompassed an air cavity. The susceptibility-matched endorectal coil uses Ultem ${ }^{\mathrm{TM}}$ as a solid support for the double loop structure of the coil, thus suppressing this air cavity. The endorectal coil used is a surface coil. The coil is composed of two rectangular loops in series, as shown in Figure 1. This design was chosen to optimize the radial uniformity of the coil, while keeping a similar SNR (compared with a single rectangular loop) ${ }^{24}$. This RF coil is a single-channel receiver coil similar in design to a double-channel receiver coil with two rectangular loops in geometrical quadrature ${ }^{25}$. Tuning and matching of the coil were performed with a serial capacitive tuning and matching circuit using capacitors (ATC 100A), and active decoupling was performed with a PIN diode (Temex 80106) placed in parallel with the coil, which detunes the coil during the transmit phase.

The performance of the coils was first assessed by quality factors $Q_{L}$ measured on the bench with a network analyzer E5071C ENA Network Analyzer (Agilent Technologies, Santa Clara, CA, USA). The loaded quality factor $Q_{L}$ was measured: The endorectal coil was inserted in a tube placed inside a small bottle of saline water with $4.5 \mathrm{~g}$ of salt per liter of distilled water (phantom 3), creating losses similar to those obtained with a larger water phantom. For both endorectal coils, the quality factor was measured with this same phantom. Bench tests were performed with and without RF traps, while keeping the total cable length at $175 \mathrm{~cm}$.

Then, to assess the relevance of using a susceptibility-matched material as a support for the endorectal coil, single-voxel PRESS acquisitions were performed to compare the FWHM of proton 
MR spectra obtained with the standard and susceptibility-matched endorectal coil. The endorectal coils were inserted in the central hole of a cylindrical phantom previously used for the comparison of susceptibility-matched material (Figure 3).

Furthermore, for examination of the rectum, the patient is usually in supine position and thus the lumen (until the left colonic flexure) defines a small angle with the $B_{0}$ magnetic field. Therefore, PRESS acquisitions were performed in the xy plane with $0^{\circ}, 15^{\circ}, 30^{\circ}$, and $45^{\circ}$ angulations between the phantom axis and the $B_{0}$ magnetic field direction. The pre-scan parameters (transmit and receive gains, central frequency and shim values) were defined with the endorectal coil aligned with $\mathrm{B}_{0}$ and then kept identical throughout the experiments. The NMR tubes contained choline or creatine solutions at $50 \mathrm{mmol} / \mathrm{L}$.

For the first series of data, the voxel was prescribed on a small volume of $4 \times 4 \times 4 \mathrm{~mm}^{3}$ (the minimum volume that can be prescribed with a standard PRESS sequence on the MR scanner in clinical mode) inside an NMR tube (Figure 4a). For the second series of data, the prescription of the voxel was realized on a larger volume of $10 \times 24.8 \times 25.5 \mathrm{~mm}^{3}$ including the endorectal coil (susceptibility-matched or not) and two NMR tubes containing metabolite solutions. In both cases, the other parameters were: TE/TR $=35 \mathrm{~ms} / 1800 \mathrm{~ms}$, spectral bandwidth $=5 \mathrm{kHz}$, number of sampling points $=4096$, number of accumulations $=128$, and scan duration $=230.4 \mathrm{~s}$ (Figure $4 \mathrm{~b}$ ). For the choline and creatine singlets, the baseline level was computed as the mean of the signal over a definite region of the spectrum [-2ppm; -1ppm] where there are no metabolites. The FWHM was then determined to be insensitive to baseline suppression errors.

Finally, to assess the relevance of using an endorectal coil, the SNR of the susceptibility-matched endorectal coil was compared with the SNR of a clinical 32-channel surface coil. A cubic phantom containing water with $4.5 \mathrm{~g}$ of salt per liter of distilled water and doped with gadolinium at 0.57 $\mathrm{mmol} / \mathrm{L}$, in order to reduce the T1-value (phantom 4, Figure 8), was used for SNR comparison. First, SNR profiles were acquired with proton density-weighted FSE-XL imaging. Then the SNR of a singlevoxel PRESS acquisition was compared for both the susceptibility-matched endorectal coil and the 32-channel torso array coil. The endorectal coil was able to acquire a spectrum on a restricted volume $4 \times 4 \times 4 \mathrm{~mm}^{3}$ unlike the 32 -channel coil, which was simply not sensitive enough. Therefore, in order to gain a sufficient SNR for the 32-channel coil, the spectra had to be acquired on a larger 
1 volume: $10 \times 10 \times 10 \mathrm{~mm}^{3}$. The normalization of SNR with volume allows the comparison to be made, 2 even though acquisitions were performed on different volumes.

3 


\section{Results}

Without any RF heating suppression circuits, the maximum increase in temperature was $7{ }^{\circ} \mathrm{C}$ (Figure 5a). The introduction of five RF traps decreased it to $0.4^{\circ} \mathrm{C}$ for a cable length of $310 \mathrm{~cm}$. Thus RF heating was below the authorized level in clinical mode, which is $1{ }^{\circ} \mathrm{C} \underline{\mathbf{2 6}}$. Furthermore, local SAR estimates indicate that for five RF traps (Table 1, Figure $5 \mathrm{~b}$ ), the maximal local SAR is $5 \mathrm{~W} / \mathrm{kg}$ and below the IEC-60601 standard, which requires an SAR of $<10 \mathrm{~W} / \mathrm{kg}$ estimated over a $10-\mathrm{g}$ mass. On MR images acquired with the fast-spin echo images, the incorporation of five RF traps suppressed the black band artifacts marking the RF field non-uniformity (Figure $5 c, d$ ) and restored the expected coil sensitivity pattern.

As expected, visible image distortions occurred in the presence of air-water interfaces. These distortions appeared as arrowhead shapes on fast-spin echo images and as irregular shapes on gradient echo images (at location 3 in Figure 6) $\underline{\mathbf{2 7}}$ and demonstrate the need for a susceptibilitymatched material. Barium sulfate created a slight visible distortion on both fast-spin echo and gradient echo images. PFC and Ultem ${ }^{\mathrm{TM}}$ did not produce any visible distortion, neither on fast-spin echo nor on gradient echo images.

The comparison plots of spectra obtained on two metabolites showed a similar SNR for both coils (Figure 7), which was already expected from the bench tests results. The loaded quality factor $Q_{L}$ achieved with the endorectal coil was 88 and dropped to 84 with the adjunction of five RF traps. With the endorectal coil built with Ultem, the $Q_{L}$ achieved was 100 and dropped to 91 with the adjunction of five RF traps.

For both RF coils, with and without Ultem, the insertion of five RF traps created a slight $4 \%$ and $10 \%$ decrease in the quality factor, respectively. Furthermore, a reduced linewidth was obtained for an endorectal coil using Ultem compared with an endorectal coil of the same geometry but encompassing an air cavity. These reduced linewidths were observed for both metabolites tested (choline and creatine). For a $0^{\circ}$ angulation, FWHM was decreased by using the susceptibilitymatched endorectal coil: by $28 \%$ for choline and $38 \%$ for creatine. For a $15^{\circ}$ angulation, FWHM was decreased by $37 \%$ for choline and $49.7 \%$ for creatine (Table 2 ). MR images acquired with a susceptibility-matched endorectal coil showed no distortions despite parameters being set to maximize the magnetic susceptibility artifact. 
1 The SNR of the 32-channel surface coil was uniform across the FOV whereas the SNR of the 2 endorectal coil decreased with distance. The SNR profiles showed a superior SNR for the endorectal 3 coil for a distance between 0 and $3.5 \mathrm{~cm}$ from the center of the endorectal coil. In spectroscopy, the 4 volume-normalized SNR was between 3 and 7 times greater using the endorectal coil (examples of 5 spectrum in Figure 7). These results are consistent with the SNR derived from the image sensitivity 6 profiles with the distance: at $1 \mathrm{~cm}$, the SNR of the endorectal coil was around 400 and the SNR of 7 the 32-channel coil was between 50 and 75, yielding a ratio between 5 and 7.8 (Figure 8). 


\section{Discussion}

In the present study, the safety of the endorectal coil was ensured with the incorporation of several RF traps. Indeed, without any RF traps, significant heating (up to $7{ }^{\circ} \mathrm{C}$ ) occurred. In addition, the incorporation of five passive RF traps along the cable connecting the endorectal coil to the scanner enabled efficient RF heating reduction.

Based on these temperature measurements, the local SAR estimates were found to be inferior to the required level and lend confidence to the assumption that no significant heating may occur. This method of local SAR estimation does not account for the third dimension, which should not be a problem since the agar-agar gel phantom may be considered homogeneous. However, the impact of the presence of the coil may modify the simulated temperature distribution. It was therefore important to conduct local 3D SAR simulations that also confirmed the location of hot spots for this specific case of elongated planar endorectal coil.. The results confirmed that hot spots are located at proximal and distal extremities, verifying that optical fiber sensor was accurately positioned to measure the local temperature. The temperature measurements were conducted with a $310-\mathrm{cm}$ long reception cable. A shorter cable would imply inferior RF heating and would not allow us to measure the effect of each trap on the temperature decrease.

The size of the RF traps was also kept to a minimum (outer diameter below $7 \mathrm{~mm}$ ) to allow for an easy insertion through the rectum of the patient.

The RF traps made it possible to experimentally minimize the RF field non-uniformity (Figure $5 c$, d). From a physical standpoint, this artifact is due to the standing wave (sheath currents) occurring in the reception cable and manifested by black curves disrupting the sensitivity of the coil. Electromagnetic simulations are not the main focus of this article but these could be performed to provide a theoretical confirmation of this phenomenon.

The strategy of incorporating several RF traps in the galvanic connection between the coil and the scanner is not the only one that can be adopted to reduce the electric field artifact, and some ongoing studies have assessed the feasibility of an optical connection both for the NMR signal received and for the active optical decoupling of the reception coil. This strategy would directly suppress the electric field artifacts, but at the cost of greater design complexity ${ }^{28,29}$. 
Our experiments confirmed that a susceptibility-matched endorectal coil significantly enhanced the ability to acquire non-distorted images whether using fast-spin echo or gradient echo sequences. It should be noted that several studies have shown that Ultem may have a larger signal distribution at higher static magnetic field, $4.7 \mathrm{~T}^{30}$ and $9.4 \mathrm{~T}^{31}$. However, at $3 \mathrm{~T}$, PFC and Ultem ${ }^{\mathrm{TM}}$ were found to be superior to barium sulfate $100 \% \mathrm{w} / \mathrm{v}$ since they yielded no visible distortion and no signal from these materials could be observed, thus avoiding unwanted signal in the regions immediately surrounding the endorectal coil. Therefore, Ultem ${ }^{\mathrm{TM}}$ has comparable performance to PFC and is superior to barium sulfate using both FSE-XL and GRE sequences. However, because we need to integrate the susceptibility-matched material inside the coil, Ultem ${ }^{\mathrm{TM}}$ has the extra advantage of being a solid and a machinable plastic. PFC, on the contrary, is a liquid and requires a more complex integration with the coil with possible liquid leakage.

This absence of image distortion indicates a better $B_{0}$ homogeneity, which was confirmed by the MRS acquisitions. For every metabolite, the spectra acquired with the susceptibility-matched endorectal coil showed a consistent decrease in the FWHM ( 30\%) compared with the original endorectal coil, and this tendency was confirmed for small angulations of the phantom. As expected, improved $B_{0}$ homogeneity involves a narrower linewidth with less potential overlap. The quantitative values of the FWHM were found to be inferior to those obtained in the literature ${ }^{17,32}$; however, the latter values were obtained in vivo, unlike the present work performed on phantoms.

It has been shown that colorectal cancer produces metabolic changes that can be seen on spectra ${ }^{33}$. This makes MRS an interesting modality for noninvasive chemical analysis of local tumors and may avoid the systematic use of histology studies. For example, choline and citrate metabolites are pertinent biomarkers of prostate cancer ${ }^{34}$. Thus, the improved quality of the spectra obtained with susceptibility-matched materials seems very promising. Besides, compared with the SNR obtained with a 32-channel torso coil, which is often used in the examination of the abdomen and rectal wall ${ }^{35,36}$ for rectal cancer staging, the SNR of the endorectal coil is very significantly superior within a radius of $3.5 \mathrm{~cm}$, corresponding to the targeted region of interest. This would be relevant for in vivo MRI and even more so in MRS ${ }^{37}$ where in vivo metabolite concentrations are lower than in the phantom used. Hence, the extra SNR could be used to either reduce the voxel size or to reduce the acquisition time. 
1 In conclusion, Ultem ${ }^{\mathrm{TM}}$ is a suitable susceptibility-matched material for endorectal coils: It can 2 significantly cancel magnetic susceptibility artifacts at the coil-tissue interface, it does not depict 3 any visible proton signal, and is a solid material that can be used as support for loop conductors. 4 With the adjunction of five RF traps located at regular intervals along the reception cable, RF heating 5 is reduced below authorized levels and the coil sensitivity profile is restored. The comparison with 6 a 32-channel array coil demonstrated a superior SNR in a 3.5-cm radius around the endorectal coil. 7 Since the rectum tightens around the endorectal coil, the rectal wall would be located at 8 approximately $4-5 \mathrm{~mm}$ from the center of the endorectal coil. It will thus be possible to achieve a 9 significantly smaller volume of examination in the rectal or colonic wall close to the lumen with an 10 adequate SNR. 
$1 \quad$ Acknowledgments:

2 This work was co-funded by the ANRT and General Electric Healthcare (General Electric 3 Healthcare, Milwaukee, WI, USA) and performed within the framework of the LABEX PRIMES (ANR4 11-LABX-0063) of Université de Lyon, within the program "Investissements d'Avenir" (ANR-11-IDEX5 0007) run by the French National Research Agency (ANR). 


\section{References}

1. Costa-Silva L, Brown G. Magnetic Resonance Imaging of Rectal Cancer. Magn Reson Imaging Clin N Am. 2013;21(2):385-408. doi:10.1016/j.mric.2013.01.006

2. Torricelli P. Rectal cancer staging. Surg Oncol. 2007;16:49-50. doi:10.1016/j.suronc.2007.10.037

3. Stoker J, Rociu E, Zwamborn AW, Schouten WR, Laméris JS. Endoluminal MR Imaging of the Rectum and Anus: Technique, Applications, and Pitfalls. RadioGraphics. 1999;19(2):383-398. doi:10.1148/radiographics.19.2.g99mr01383

4. Dempsey MF, Condon B, Hadley DM. Investigation of the factors responsible for burns during MRI. J Magn Reson Imaging. 2001;13(4):627-631. doi:10.1002/jmri.1088

5. Armenean C, Beuf $O$, Armenean M, Pilleul F, Perrin E, Saint-Jalmes H. IRM Interventionnelle : Risques de brûlure du patient et du radiologue. J Radiol. 2007;88(4):599-600. doi:10.1016/S02210363(07)89863-5

6. Konings MK, Bartels LW, Smits HFM, Bakker CJG. Heating Around Intravascular Guidewires by Resonating RF Waves. J Magn Reson Imaging. 2000;12(1):79-85. doi:10.1002/15222586(200007)12:1<79::AID-JMRI9>3.0.CO;2-T

7. Harrison $\mathrm{WH}$, Arakawa M, McCarten BM. RF coil coupling for MRI with tuned RF rejection circuit using coax shield choke. Published online July 21, 1987. http://www.google.com/patents/US4682125

8. Wynn T, Molyneaux D. Tunable resonant cable trap. Published online December 28, 2006. Accessed August 28, 2019. https://patents.google.com/patent/US20060290448A1/en

9. Seeber DA, Jevtic J, Menon A. Floating shield current suppression trap. Concepts Magn Reson Part $B$ Magn Reson Eng. 2004;21B(1):26-31. doi:10.1002/cmr.b.20008

10. Zanchi MG, Pauly JM, Scott GC. Feasibility of Active Cable Trap to Attenuate MRI-Induced RF Currents. Proc Int/ Soc Mag Reson Med 17. Published online 2009:1.

11. Peterson DM, Beck BL, Duensing GR, Fitzsimmons JR. Common mode signal rejection methods for MRI: Reduction of cable shield currents for high static magnetic field systems. Concepts Magn Reson Part $B$ Magn Reson Eng. 2003;19B(1):1-8. doi:10.1002/cmr.b.10090

12. Heerschap A, Er GJJ. In Vivo Proton MR Spectroscopy Reveals Altered Metabolite Content in Malignant Prostate Tissue. :7.

13. Scheenen TWJ, Rosenkrantz AB, Haider MA, Fütterer JJ. Multiparametric Magnetic Resonance Imaging in Prostate Cancer Management: Current Status and Future Perspectives. Invest Radiol. 2015;50(9):594-600. doi:10.1097/RLI.0000000000000163

14. Dorez $\mathrm{H}$, Ratiney $\mathrm{H}$, Canaple $\mathrm{L}$, et al. In vivo MRS for the assessment of mouse colon using a dedicated endorectal coil: initial findings. NMR Biomed. 2017;30(12):e3794. doi:10.1002/nbm.3794

15. Dwarkasing RS, Schouten WR, Geeraedts TEA, Mitalas LE, Hop WCJ, Krestin GP. Chronic Anal and Perianal Pain Resolved With MRI. Am J Roentgenol. 2013;200(5):1034-1041. doi:10.2214/AJR.12.8813 
16. Powell DK, Kodsi KL, Levin G, Yim A, Nicholson D, Kagen AC. Comparison of comfort and image quality with two endorectal coils in MRI of the prostate. J Magn Reson Imaging. 2014;39(2):419-426. doi:10.1002/jmri.24179

17. Lee GC, Goodwill PW, Phuong K, et al. Pyrolytic graphite foam: A passive magnetic susceptibility matching material. J Magn Reson Imaging. 2010;32(3):684-691. doi:10.1002/jmri.22270

18. Rosen Y, Bloch BN, Lenkinski RE, Greenman RL, Marquis RP, Rofsky NM. 3T MR of the prostate: Reducing susceptibility gradients by inflating the endorectal coil with a barium sulfate suspension. Magn Reson Med. 2007;57(5):898-904. doi:10.1002/mrm.21166

19. Pilleul F, Saint-Jalmes H, Beuf O, et al. Sonde endocavitaire pour l'imagerie et/ou la spectrometrie par resonance magnetique nucleaire. Published online July 2, 2009. Accessed August 28, 2019. https://patents.google.com/patent/WO2009080972A2/fr

20. Verret J-M, Pilleul F, Rabrait C, et al. Characterization of a dedicated double loop, endoluminal coil for anal sphincter MR imaging at $1.5 \mathrm{~T}$ and $3 \mathrm{~T}$. Concepts Magn Reson Part B Magn Reson Eng. 2014;44(2):39-49. doi:10.1002/cmr.b.21265

21. Armenean C, Perrin E, Armenean M, Beuf O, Pilleul F, Saint-Jalmes H. RF-induced temperature elevation along metallic wires in clinical magnetic resonance imaging: Influence of diameter and length. Magn Reson Med. 2004;52(5):1200-1206. doi:10.1002/mrm.20246

22. Yeung CJ, Susil RC, Atalar E. RF safety of wires in interventional MRI: Using a safety index. Magn Reson Med. 2002;47(1):187-193. doi:10.1002/mrm.10037

23. Shrivastava D, Vaughan JT. A Generic Bioheat Transfer Thermal Model for a Perfused Tissue. J Biomech Eng. 2009;131(7). doi:10.1115/1.3127260

24. Schenck JF. The role of magnetic susceptibility in magnetic resonance imaging: MRI magnetic compatibility of the first and second kinds. Med Phys. 1996;23(6):815-850. doi:10.1118/1.597854

25. Armenean M, Beuf O, Pilleul F, Saint-Jalmes H. Optimization of endoluminal loop radiofrequency coils for gastrointestinal wall MR imaging. IEEE Sens J. 2004;4(1):57-64. doi:10.1109/JSEN.2003.820334

26. Quick HH, Ladd ME, Zimmermann-Paul GG, et al. Single-loop coil concepts for intravascular magnetic resonance imaging. Magn Reson Med. 1999;41(4):751-758. doi:10.1002/(SICI)15222594(199904)41:4<751::AID-MRM14>3.0.CO;2-V

27. Dybdahl K, International Electrical Commission System for Conformity Testing and Certification of Electrotechnical Equipment and Components (IECEE). Patient protection at risk in IEC 60601-1 3rd edition. Med Device Technol. 2009;20(5):26, 28-29.

28. Beuf $O$, Briguet $A$, Lissac M, Davis R. Magnetic Resonance Imaging for the Determination of Magnetic Susceptibility of Materials. J Magn Reson B. 1996;112(2):111-118. doi:10.1006/jmrb.1996.0120

29. Ayde R, Gaborit G, Jarrige P, et al. Potentialities of an Electro-Optic Crystal Fed by Nuclear Magnetic Resonant Resonant Coil for Remote and Low-Invasive Magnetic Field Characterization. IEEE Sens J. 2013;13(4):1274-1280. doi:10.1109/JSEN.2012.2230623 
30. Saniour I, Aydé R, Perrier AL, et al. Active optical-based detuning circuit for receiver endoluminal coil. Biomed Phys Eng Express. 2017;3(2):025002. doi:10.1088/2057-1976/aa5db0

31. Marjanska M, Waks M, Snyder CJ, Vaughan JT. Multinuclear NMR investigation of probe construction materials at 9.4T. Magn Reson Med. 2008;59(4):936-938. doi:10.1002/mrm.21566

32. Babcock EE, Vaughan JT, And B Lesan, Nunnally RL. Multinuclear nmr investigations of probe construction materials at 4.7 t. Magn Reson Med. 1990;13(3):498-503. doi:10.1002/mrm.1910130317

33. Choi H, Ma J. Use of Perfluorocarbon Compound in the Endorectal Coil to Improve MR Spectroscopy of the Prostate. Am J Roentgenol. 2008;190(4):1055-1059. doi:10.2214/AJR.07.2998

34. Duarte IF, Gil AM. Metabolic signatures of cancer unveiled by NMR spectroscopy of human biofluids. Prog Nucl Magn Reson Spectrosc. 2012;62:51-74. doi:10.1016/j.pnmrs.2011.11.002

35. Kurhanewicz J, Vigneron D, Carroll P, Coakley F. Multiparametric magnetic resonance imaging in prostate cancer: present and future. Curr Opin Urol. 2008;18(1):71-77. doi:10.1097/MOU.0b013e3282f19d01

36. Roach SC, Hulse PA, Moulding FJ, Wilson R, Carrington BM. Magnetic resonance imaging of anal cancer. Clin Radiol. 2005;60(10):1111-1119. doi:10.1016/j.crad.2005.05.008

37. Beaumont C, Pandey T, Fricke RG, Laryea J, Jambhekar K. MR Evaluation of Rectal Cancer: Current Concepts. Curr Probl Diagn Radiol. 2013;42(3):99-112. doi:10.1067/j.cpradiol.2012.08.002

\section{Tables}

Table 1. Temperature rise $\Delta T$ as a function of the number of RF traps (based on give measurements) and subsequent SAR estimations. $\Delta T$ is the difference between the final temperature, at the end of the FIESTA sequence, and the initial temperature, at the start of the FIESTA sequence.

\begin{tabular}{|l|l|l|l|l|l|l|}
\hline Number of traps & \multicolumn{1}{|c|}{0} & 1 & 2 & 3 & 4 & 5 \\
\hline $\begin{array}{l}\text { Temperature increase } \Delta \mathrm{T} \text { of } \\
\text { miniaturized RF traps }\left({ }^{\circ} \mathrm{C}\right)\end{array}$ & $7.0 \pm 0.3$ & $1.77 \pm 0.15$ & $1.64 \pm 0.03$ & $1.55 \pm 0.09$ & $1.38 \pm 0.05$ & $0.40 \pm 0.06$ \\
\hline SAR estimations $(\mathrm{W} / \mathrm{kg})$ & 80 & 20 & 19 & 18 & 16 & 5 \\
\hline
\end{tabular}




\begin{tabular}{|l|l|l|l|}
\hline $\begin{array}{l}\text { Angle } \\
\text { (degree) }\end{array}$ & Endorectal coil type & Choline FWHM $(\mathrm{Hz})$ & Creatine FWHM (Hz) \\
\hline 0 & Susceptibility matched & $3.6 \pm 0.1$ & $3.7 \pm 0.2$ \\
\cline { 2 - 4 } & Reference & $5.0 \pm 0.1$ & $6.0 \pm 0.2$ \\
\hline 15 & Susceptibility matched & 6.1 & 6.3 \\
\cline { 2 - 4 } & Reference & 9.6 & 12.7 \\
\hline
\end{tabular}

1 